\title{
El uso de rayos $X$ en odontología y la importancia de la justificación de exámenes radiográficos
}

\section{Use of $X$ rays in dentistry and the importance of justification of radiographic examinations}

\author{
L. Barba Ramírez*, V. Ruiz García de Chacón**, A. Hidalgo Rivas***
}

\section{RESUMEN}

Introducción: Los exámenes radiográficos odontológicos usan radiaciones ionizantes, las que pueden producir efectos adversos en la salud de pacientes y trabajadores ocupacionalmente expuestos a radiaciones. La protección radiológica busca que la justificación de la exposición a radiación ionizante sea adecuada, y produzca al paciente mayor beneficio que riesgo. El objetivo del presente artículo es revisar los efectos adversos de las radiaciones ionizantes en el ser humano, analizar el principio de justificación y entregar recomendaciones para su aplicación en la práctica diaria odontológica, presentando guías clínicas actuales. Revisión: Para cumplir con el principio de justificación, la elección de cada examen radiográfico debe realizarse responsablemente, basándose en las necesidades individuales de cada paciente. Este proceso debe realizarse conjuntamente entre clínico y radiólogo maxilofacial. A pesar de que existen numerosas guías para distintas especialidades odontológicas, desarrolladas por instituciones de diversas partes del mundo, son pocas las guías basadas en evidencia. Las guías aportan recomendaciones, con distintos niveles de evidencia, para el uso de exámenes radiográficos, permitiendo al clínico seleccionar el examen adecuado para cada paciente, contribuyendo a la disminución de exposiciones innecesarias.

Conclusiones: La comunicación entre clínico y radiólogo maxilofacial es fundamental para lograr el proceso de justificación, y por lo tanto, una reducción en las dosis de radiación a los pacientes. Además, es necesario el uso de guías clínicas para correcta selección del examen radiográfico. Se requiere más investigación que justifique el uso de nuevas tecnologías imagenológicas en indicaciones específicas y permitan elaborar guías clínicas basadas en evidencia.

PALABRAS CLAVE: Protección radiológica; radiología; radiografía dental; tomografía computarizada de haz cónico; guías.

\section{ABSTRACT}

Introduction: Dental radiographic examinations use ionizing radiation, which can have adverse health effects in patients and workers occupationally exposed to radiation. Radiological protection seeks to ensure that justification

* $\quad$ Docente Facultad de Odontología, Universidad de Costa Rica, Costa Rica. Especialista en Radiología Oral y Maxilofacial. Estudiante Maestría en Radiología Oral y Maxilofacial. Universidad Peruana Cayetano Heredia, Perú.

* * Docente Facultad de Estomatología, Universidad Peruana Cayetano Heredia, Perú. Especialista en Radiología Oral y Maxilofacial.

*** Docente Departamento de Estomatología, Universidad de Talca, Chile. Especialista en Radiología Oral y Maxilofacial. Doctor en Radiología Oral. 
for exposure to ionizing radiation is adequate, so that the patient has a greater benefit than risk. The aim of this article is to review the adverse effects of radiation on humans, to analyze the principle of justification and to provide recommendations for its application in daily dental practice, presenting current clinical guidelines.

Review: In order to comply with the principle of justification, the choice of each radiographic examination must be made in a responsible manner, based on the individual needs of each patient. This process should be performed jointly between the clinician and the maxillofacial radiologist. Although there are numerous guidelines for different dental specialties, developed by institutions from different parts of the world, few of them are evidence-based guidelines. Guidelines provide recommendations, with different levels of evidence, for the use of radiographic examination, allowing the clinician to select the appropriate exam for each patient, contributing to the reduction of unnecessary exposures.

Conclusions: Communication between clinician and maxillofacial radiologist is essential to achieve the justification process, and therefore a reduction in radiation doses to patients. In addition, the use of clinical guidelines is necessary for a correct choice of the radiographic examination. More research is needed to justify the use of new imaging technologies in specific indications, to develop clinical evidence-based guidelines.

KEY WORDS: Radiation protection; radiology; dental radiography; cone-beam computed tomography; guidelines.

Fecha de recepción: 9 de junio de 2019

Fecha de aceptación: 4 de septiembre de 2019

L. Barba Ramírez, V. Ruiz García de Chacón, A. Hidalgo Rivas. El uso de rayos X en odontología y la importancia de la justificación de exámenes radiográficos . 2020; 36, (3): 131-142.

\section{INTRODUCCIÓN}

Los exámenes radiográficos son una herramienta que aporta información al odontólogo, y que junto con el examen clínico, ayudan a un correcto diagnóstico y tratamiento del paciente. En odontología, tanto en los exámenes radiográficos convencionales bidimensionales (2D) como en los tridimensionales (3D) como la tomografía computarizada de haz cónico (TCHC), se expone al paciente a rayos $\mathrm{X}$, y esta exposición puede producir efectos dañinos. Esto, debido a que incluso dosis bajas como las usadas en odontología, podrían producir efectos nocivos en la salud.(1) Estos efectos, conocidos como estocásticos, son el cáncer y los efectos hereditarios. ${ }^{(2)}$

Debido al riesgo inherente que presenta la exposición a radiaciones ionizantes, la prescripción de cada examen radiográfico debe estar justificada. Es decir, debe elegirse el examen más adecuado para la necesidad del paciente, de manera que el paciente obtenga un beneficio mayor que el riesgo al que se expone. ${ }^{(1)}$ El proceso de justificación del examen radiográfico debe realizarse conjuntamente entre el radiólogo maxilofacial y el clínico, y debiera estar basado en evidencia. ${ }^{(2)}$ El radiólogo maxilofacial, por su formación especializada en imagenología, tiene el conocimiento necesario para cooperar con la justificación de los exámenes radiográficos.

Numerosas publicaciones tratan sobre los principios de protección radiológica ${ }^{(1,3,4)}$ y múltiples guías orientan en la prescripción de exámenes radiográficos. ${ }^{(5-9)}$ Estos documentos fundamentan y sirven de ayuda en el proceso de justificación de exámenes radiográficos. Se ha descrito también un modelo que contribuye en la selección de exámenes imagenológicos, ${ }^{(10)}$ al evaluar la utilidad de éstos en el proceso de toma de decisiones en beneficio al paciente. El uso de este modelo es mayor en medicina, con poca aplicación en odontología. No se encontró publicaciones que recopilen e integren esta información en odontología, llevándola a la práctica diaria.

El propósito del presente artículo es revisar los efectos adversos de las radiaciones ionizantes en el ser humano, analizar el principio de justificación y entregar recomendaciones para su aplicación en la práctica diaria odontológica, presentando las guías clínicas actuales.

\section{LOS RAYOS X Y EL FENÓMENO DE IONIZACIÓN}

Los rayos $\mathrm{X}$ son un tipo de radiación del espectro electromagnético, de onda muy corta y alta frecuencia, que tiene la capacidad de producir ioni- 
zación en los átomos de la materia en que incide. La ionización es el fenómeno por el que los rayos $\mathrm{X}$, al golpear un átomo, remueve un electrón de los orbitales internos, provocando que el átomo se convierta en un ion positivo y el electrón removido en un ion negativo. ${ }^{(6,11)}$ En el espectro de radiación electromagnética se incluyen otros tipos de radiación como los rayos gamma $(Y)$, rayos ultravioleta, luz visible, radiación infrarroja, microondas y ondas de radio. De éstas, solamente la radiación ultravioleta, los rayos $\mathbf{X}$ y $\mathbf{Y}$ son capaces de producir ionización. A estas radiaciones que producen ionización se les conoce como radiaciones ionizantes.

Las fuentes de radiación ionizante pueden ser naturales o artificiales. Las fuentes de radiación artificial son las exposiciones médicas y ocupacionales. ${ }^{(12)}$ Entre las exposiciones médicas se encuentra la exposición del paciente a radiaciones ionizantes durante el tratamiento odontológico. Pese a que en odontología las dosis de radiación usadas son relativamente bajas, ${ }^{(5)}$ es común que los pacientes sean expuestos a rayos $\mathrm{X}$ repetidamente durante sus tratamientos, y a lo largo de su vida. ${ }^{(12)}$. Esto aumenta las posibilidades del paciente de desarrollar algún efecto adverso por radiaciones ionizantes, ya que las dosis de radiación son acumulativas. ${ }^{(1)}$

\section{EFECTOS DE LAS RADIACIONES IONIZANTES}

Los efectos de las radiaciones ionizantes comenzaron a observarse poco tiempo después de que W.C. Roentgen descubriera los rayos X en 1895. (13) Son muchos los que murieron por exposición a rayos $\mathrm{X}$ en los primeros años de la radiología. (14) En ese tiempo las exposiciones a rayos X podían ir desde 20 minutos y extenderse por horas, causando daños en los tejidos irradiados, tales como pérdida de cabello, carcinomas y muerte. (14) Pese a que las dosis de radiación en los primeros años de uso de los rayos X era mucho mayor a la usada actualmente en odontología, la dosis de radiación recibida por el paciente sigue siendo de importancia.

Los efectos de las radiaciones ionizantes en la salud se producen debido al daño en los átomos de las moléculas provocado por la ionización. ${ }^{(6,11)} \mathrm{Si}$ el daño provocado por la ionización no es reparado, la célula afectada puede morir, o puede sobrevivir pero con una alteración en su genoma. ${ }^{(1,6,15)} \mathrm{Am}$ bas situaciones pueden producir efectos adversos para la salud. Estos efectos se clasifican en dos categorías: reacciones tisulares (antes llamados efectos determinísticos) y efectos estocásticos. ${ }^{(2,3)}$

\section{Reacciones Tisulares y Efectos Estocásticos}

Las reacciones tisulares se presentan cuando muere un número elevado de células irradiadas, produciendo un daño observable, manifestado como una pérdida de funcionalidad del tejido. ${ }^{(1)}$ Estas reacciones tienen un umbral de dosis de radiación bajo el cual no se produce el efecto, y a medida que se supera el umbral, la severidad de la reacción aumenta. Así, la severidad de la reacción es dependiente del total de dosis de radiación recibida por el órgano. ${ }^{(2)}$ Las reacciones tisulares ocurren a dosis altas, sobre los 100 milisievert (mSv), en una sola dosis o como dosis anual, ${ }^{(1)}$ siendo estas dosis habitualmente muy por sobre las utilizadas en odontología. ${ }^{(6)}$ Las reacciones tisulares pueden manifestarse de manera temprana (horas o pocas semanas) e incluyen eritema, mucositis, pérdida de cabello, entre otros; y las reacciones tisulares tardías (meses a años) incluyen cataratas, ulceración mucosa severa entre otros. ${ }^{(1,2,16)}$

Los efectos estocásticos se presentan cuando la célula irradiada o un pequeño número de células, sobrevive con una alteración en su genoma. Estos efectos no tienen umbral de dosis, por lo que aún dosis mínimas de radiación ionizante conllevan la probabilidad de producirlos, ${ }^{(2)}$ y a medida que aumenta la dosis, aumenta la probabilidad de que se presenten estos efectos, no así la severidad de éstos. (2) Los efectos estocásticos se manifiestan de manera tardía, y el periodo latente entre la exposición a los rayos $\mathrm{X}$ y el diagnóstico clínico de un tumor puede ser de muchos años. ${ }^{(6)}$ Si la alteración en el genoma se produce en células germinales, el efecto estocástico se expresa como un efecto hereditario, y si se produce en células somáticas se expresa como un cáncer. ${ }^{(2,5)}$

Debido a que en odontología las dosis de radiación son bajas y no debieran exceder los $100 \mathrm{mSv}$ por año, los efectos determinísticos suelen no presentarse, ${ }^{(1,4)}$ siendo los efectos que se podrían presentar los estocásticos. Entre los efectos estocásticos, el riesgo principal en odontología es la inducción de cáncer. ${ }^{(1)}$ Los pacientes pediátricos tienen mayor riesgo de inducción de cáncer por radiación ionizante que los pacientes adultos. ${ }^{(17)}$ Esto se debe a que sus órganos en desarrollo son 
más radiosensibles y a su mayor expectativa de vida, siendo las niñas significativamente más radiosensibles que los niños. ${ }^{(17)}$

\section{Modelo Lineal sin UMBRAL}

Existen distintos modelos que explican la relación entre las bajas dosis de radiación ionizante y sus efectos adversos. ${ }^{(18)}$ La Comisión Internacional de Protección Radiológica (ICRP del inglés International Commission on Radiological Protection) avala el modelo lineal sin umbral (LNT del inglés linear-no-threshold). Este modelo asume que, en el rango de dosis bajas de radiación ionizante, cualquier dosis diferente a cero aumentaría el riesgo de inducción de cáncer o efectos genéticos heredables. ${ }^{(1)}$ La ICRP sostiene que el modelo LNT es el más prudente a utilizar en relación con el uso seguro de las radiaciones ionizantes. ${ }^{(4)}$ Lo mismo señala el Consejo Nacional de Protección Radiológica y Mediciones de Estados Unidos de América (NCRP del inglés National Council on Radiation Protection and Measurements). El NCRP, luego de una revisión crítica de estudios epidemiológicos recientes de poblaciones expuestas a radiaciones ionizantes, concluyó que la mayoría de estos estudios aportan evidencia que apoya el uso del modelo LNT en protección radiológica. ${ }^{(19)}$

\section{PRINCIPIOS DE PROTECCIÓN RADIOLÓGICA}

Debido a la probabilidad de las radiaciones ionizantes de producir efectos adversos tanto en pacientes como en los trabajadores ocupacionalmente expuestos a radiaciones, la ICRP ha establecido tres principios de protección radiológica. (20) Estos principios son optimización, justificación y limitación de dosis, y deben aplicarse siempre que se trabaje con radiaciones ionizantes. ${ }^{(2)} \mathrm{Si}$ bien la presente revisión aborda sólo el principio de justificación, es conveniente definir los tres principios de protección radiológica.

\section{PRINCIPIO DE Optimización}

El principio de optimización, conocido como ALARA (del inglés As Low As Reasonably Achievable) establece que se debe utilizar la dosis de más baja radiación que entregue una imagen diagnóstica de calidad aceptable para la indicación clínica. ${ }^{(1,2,}$

5) Como alternativa a ALARA, se ha propuesto el acrónimo ALADA (del inglés As Low As Diagnostically Acceptable), con el fin de hacer énfasis en mantener la calidad diagnóstica de la radiografía. ${ }^{(21)}$
Recientemente, el proyecto DIMITRA (del inglés dentomaxillofacial paediatric imaging: an investigation towards low-dose radiation induced risks) propone una nueva visión del principio de optimización. Su propuesta busca una definición más precisa introduciendo el acrónimo ALADAIP (del inglés As Low as Diagnostically Acceptable being Indication-oriented and Patient-specific). De esta manera, agrega al acrónimo anterior, que el examen se realice de acuerdo con la indicación clínica y sea específico para el paciente, adaptando los parámetros a esta indicación y a la edad del mismo. ${ }^{(22)}$

El principio de optimización abarca los recursos disponibles para la protección de las personas, estableciendo la necesidad del uso de barreras de protección al paciente, promoviendo la selección del examen radiográfico en que los órganos radiosensibles reciban la menor dosis, y la selección de los parámetros de exposición adecuados, tales como kilovoltaje, miliamperaje y tiempo. ${ }^{(5)}$

\section{Principio de Limitación de Dosis}

El principio de limitación de dosis señala que la exposición a radiaciones ionizantes de los trabajadores ocupacionalmente expuestos a radiaciones y del público, no debe exceder los límites de dosis recomendados en un período de tiempo específico. (1) Estos límites de dosis son los recomendados por la ICRP y/o establecidos en las regulaciones nacionales. ${ }^{(1)}$ Este principio se relaciona principalmente con el blindaje de la sala de rayos $\mathrm{X}$ y el uso de biombo plomado, además del cumplimiento de la distancia mínima establecida entre la fuente de emisión de radiación y el personal y/o público. ${ }^{(2)} \mathrm{El}$ principio de limitación de dosis no se aplica a los pacientes, ${ }^{(1,2,4)}$ considerando que la limitación de dosis a un paciente podría impedir el uso de radiaciones ionizantes, aún estando justificadas para un paciente. Otra manera en que el operador cumple con el principio de limitación de dosis, es mediante una correcta aplicación de los principios de justificación y optimización con sus pacientes. ${ }^{(23)}$

\section{PRINCIPIO dE Justificación}

El principio de justificación establece que la decisión de indicar un examen que utilice radiaciones ionizantes debe producir más beneficio que el riesgo inherente a la exposición a dicha radiación. El beneficio obtenido de una exposición a radiación debe poder aplicarse tanto a los individuos 
como a la sociedad en general. ${ }^{(1)}$ La ICRP define tres niveles para la aplicación del principio de justificación. ${ }^{(1)}$ En el nivel 1, que es el más general, se acepta el uso de la radiación en medicina cuando produce más beneficio que daño al paciente. En este sentido, actualmente, se acepta que el uso de rayos $\mathrm{X}$ en medicina y odontología está justificado. En el nivel 2 se valora si se justifica un examen radiográfico específico para una indicación específica, considerando si este examen entrega información necesaria para mejorar el diagnóstico o tratamiento. En el nivel 3 se valora de modo individual, si el examen radiográfico podría tener algún impacto en el diagnóstico o en el tratamiento de un paciente en particular, estimando que el uso de dicho examen traerá más beneficio que riesgo al paciente en particular. Si el examen radiográfico no produce un cambio en el diagnóstico y/o tratamiento, que beneficie al paciente, podría no estar justificada su indicación. ${ }^{(1,2,4)}$ Es decir, cualquier exposición a radiación ionizante de cada paciente en particular, debería justificarse previamente, considerando los objetivos de la exposición y las características particulares del paciente. ${ }^{(1,5)}$

Contrario al principio de justificación, existen las llamadas radiografías de "rutina", que son indicadas con una misma periodicidad para todos los pacientes. ${ }^{(5,6)}$ Las radiografías de rutina podrían exponer a los pacientes de manera innecesaria a la radiación, ya que no se basan en las necesidades individuales de los pacientes y no toman en cuenta la presencia o ausencia de signos y síntomas de cada paciente. ${ }^{(6)}$ Es por esto que el uso de radiografías de rutina se contraindica como examen radiográfico en odontología. ${ }^{(5,6)}$

\section{APLICACIÓN DEL PRINCIPIO DE JUSTIFICACIÓN}

\section{DE EXÁMENES RADIOGRÁFICOS EN ODONTOLOGÍA}

Para aplicar correctamente el principio de justificación de exámenes radiográficos es fundamental la comunicación entre el clínico y el radiólogo maxilofacial, ${ }^{(2)}$ y de esta manera, realizar el examen radiográfico más indicado, de acuerdo con la necesidad y en beneficio de la salud del paciente. ${ }^{(2,5)}$

En relación con el rol del clínico en el proceso de justificación de exámenes radiográficos, éste debe realizar una completa historia clínica y médica, y un exhaustivo examen intraoral. El clínico además debe considerar la prevalencia e incidencia de la enfermedad que sospecha y sus factores de riesgo,
$(9,24)$ y solicitar los exámenes radiográficos previos del paciente. ${ }^{(1,2,12)}$ Solo después de esto, debe evaluar si es necesario solicitar un nuevo examen radiográfico. ${ }^{(1)}$ Si lo considera indispensable, el clínico debe solicitar el examen indicado para la necesidad de su paciente. ${ }^{(1,2,6,12)}$. Al solicitar el examen radiográfico, el clínico debe aportar la información necesaria ${ }^{(25)}$ en la solicitud radiográfica.

El rol del radiólogo maxilofacial en el proceso de justificación comienza luego que éste recibe la solicitud radiográfica del clínico. Con la información contenida en esta solicitud, el radiólogo maxilofacial aplica sus conocimientos y experiencia acerca del rendimiento de los diversos exámenes radiográficos, para determinar si el examen solicitado está correctamente justificado. En caso de no estarlo, el radiólogo maxilofacial puede comunicarse con el clínico para sugerir un examen radiográfico distinto al solicitado, pero con el rendimiento necesario para la indicación clínica. Además, el radiólogo maxilofacial puede recomendar exámenes alternativos que puedan entregar la misma información, pero con menos radiación para el paciente. ${ }^{(2,5)}$

La solicitud radiográfica es fundamental en el proceso de justificación, siendo el medio de comunicación entre el clínico y el radiólogo. $(25,26)$ El correcto llenado de la solicitud radiográfica por parte del clínico permite al radiólogo determinar la justificación del examen, al conocer los antecedentes clínicos del paciente, el propósito del examen y el diagnóstico presuntivo. ${ }^{(1,26)}$ Es importante que la información de contacto del clínico se encuentre en la solicitud radiográfica, de manera que el radiólogo pueda contactarlo en caso de que sea necesario sugerir algún cambio al examen solicitado, o resolver alguna duda. ${ }^{(27)}$ Si bien no existe un formato establecido para la solicitud radiográfica, distintos organismos internacionales han estipulado la información mínima que debiera contener la solicitud radiográfica. ${ }^{(25)}$ Esta información debiera incluir al menos el diagnóstico presuntivo, tipo de examen solicitado y lo que desea obtener del examen radiográfico.

En la actualidad, en muchas ocasiones, los equipos radiográficos se usan en centros de salud en los que no se cuenta con un radiólogo maxilofacial. La ausencia de un radiólogo maxilofacial en centros de salud podría generar exposiciones innecesarias a los pacientes con un aumento de la 
dosis recibida por ellos, debido a un proceso de justificación realizado de forma inadecuada.

Debido a la existencia de diversos exámenes radiográficos, cada uno con distintas indicaciones y limitaciones, la selección del examen imagenológico justificado para cada situación clínica, debiera basarse en su eficacia diagnóstica. Se ha descrito un modelo que contribuye a la selección del examen imagenológico según su eficacia diagnóstica. Este modelo evalúa la utilidad de los diferentes exámenes imagenológicos en el proceso de toma de decisión diagnóstica y de tratamiento por parte del clínico, de manera que el paciente obtenga un mayor beneficio del examen imagenológico que se le indica, de acuerdo con su necesidad.

\section{EFICACIA DIAGNÓSTICA DE \\ LOS EXÁMENES RADIOGRÁFICOS}

El modelo que contribuye a la selección del examen imagenológico según su eficacia diagnóstica es el modelo jerárquico de Eficacia Diagnóstica descrito por Fryback y Thornbury en el año 1991. (10) Este modelo se basa en una escala de mediciones de desempeño, clasificada en seis niveles y se utiliza para comparar la eficacia diagnóstica de un examen radiográfico usado habitualmente con una nueva tecnología imagenológica. Al comparar dos tecnologías imagenológicas para una misma indicación específica usando este modelo, se busca determinar si se justifica el uso de la nueva tecnología en beneficio del paciente. Si este beneficio produce al menos un diagnóstico más preciso o un mejor tratamiento para el paciente, se justificaría el uso de la nueva tecnología para la indicación específica.

Para que un examen radiográfico tenga eficacia diagnóstica en niveles superiores del modelo jerárquico de Fryback y Thornbury, se debe comprobar su eficacia en los niveles inferiores. Sin embargo, una mayor eficacia en un nivel inferior no garantiza eficacia en niveles superiores. Los exámenes radiográficos que presentan eficacia en niveles superiores del modelo son aquellos que representan mayor beneficio para el paciente, siendo los exámenes con eficacia en el nivel superior los que aportan beneficio a la sociedad.

Considerando que la TCHC es una tecnología relativamente reciente en odontología, su eficacia diagnóstica debe evaluarse en las distintas indicaciones para las diferentes especialidades. Así, se

TABLA 1. Clasificación de los niveles del Modelo Jerárquico de Eficacia Diagnóstica. Adaptado de Fryback y Thornbury, 1991

NIVEL

1. Eficacia Técnica

$\begin{array}{ll}\text { - } & \text { - } \text { - } 2 \text { refoluctosios. } \\ & \text {-Nitidez, brillo, contraste. }\end{array}$

2. Eficacia en la

Exactitud Diagnóstica

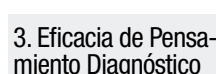

miento Diagnóstico

4. Eficacia Terapéutica

5. Eficacia en Resulta-

do para el paciente

6. Eficacia Social

\section{EJEMPLOS}

-Resolución espacia

-Cambio en la función de transferencia de modulación.

-Sensibilidad, especificidad -Valores predictivos positivos y negativos. Receiver Operating Characteristic).

-Porcentaje de casos en que la imagen se juzgó útil para realizar el diagnóstico. y después. cos debido a la información del examen.

-Porcentaje de veces en que cambio la terapia propuesta mente de los informes clínicos). del examen. ción del examen.

-Aumento en la esperanza de vida o calidad de vida.
-Porcentaje de diagnósticos correctos en una serie de casos.

-Curva ROC (Característica Operativa del Receptor, del inglés

-Cambio en la distribución del diagnóstico diferencial antes

-Diferencias en el diagnostico diferencial antes y después.

-Porcentaje de veces que la imagen se juzgó útil en el manejo 0 tratamiento del paciente.

-Porcentaje de veces que se evitaron procedimientos médiantes, tras la información del examen (inferido retrospectiva-

-Porcentaje de pacientes que mejoraron con la información del examen comparados con los pacientes sin la información

-Morbilidad o procedimientos evitados debido a la informa-

-Análisis costo-beneficio y costo-efectividad desde el punto de vista social.

justificaría su uso racional y seguro en los casos que se demuestre su mayor eficacia en comparación con el examen radiográfico habitual.

La Tabla 1 presenta la clasificación del modelo jerárquico de Eficacia Diagnóstica de Fryback y Thornbury, con ejemplos de las medidas características para cada nivel.

El modelo de Eficacia Diagnóstica de Fryback y Thornbury puede aplicarse para evaluar distintas técnicas o tecnologías imagenológicas. Al comparar la nueva técnica o tecnología con la usada habitualmente en los distintos niveles, se evalúa el resultado obtenido. Esta evaluación demuestra cuál tecnología tiene mayor eficacia diagnóstica. Si ambas tecnologías tienen el mismo resultado, estarían aportando la misma información, por lo que no se justificaría su indicación ya que la nueva técnica o tecnología no tendría beneficios adicionales para el paciente. ${ }^{(28)}$

\section{GUÍAS PARA LA PRESCRIPCIÓN DE EXÁMENES RADIOGRÁFICOS EN ODONTOLOGÍA} Como ayuda en el proceso de justificación del examen radiográfico existen una serie de guías 


\begin{tabular}{|c|c|c|c|c|c|c|c|}
\hline & & & TABLA 2 & & & & \\
\hline \multicolumn{2}{|c|}{ PUBLICACIÓN } & \multirow{2}{*}{$\begin{array}{l}\text { AUTOR } \\
\text { FGDP }\end{array}$} & \multirow{2}{*}{$\begin{array}{l}\text { AÑO DE } \\
\text { PUIBLICACIÓN } \\
2013\end{array}$} & \multirow{2}{*}{$\begin{array}{l}\text { ÁREAS CLÍNICAS } \\
\text { General }\end{array}$} & \multirow{2}{*}{$\begin{array}{l}3 D \\
x\end{array}$} & \multirow{2}{*}{$\begin{array}{l}2 \mathrm{D} \\
\mathrm{x}\end{array}$} & \multirow{2}{*}{$\begin{array}{l}\text { MÉTODO DE } \\
\text { DESARROLLO DE LA GUIIA } \\
\text { Juicio de expertos }\end{array}$} \\
\hline 1. & Selection Criteria for Dental Radiography ${ }^{(6)}$ & & & & & & \\
\hline 2 & $\begin{array}{l}\text { Radiation Protection } 172 \text {. } \\
\text { CBCT for dental and maxillofacial radiology. } \\
\text { Evidence-based guidelines (5) }\end{array}$ & $\begin{array}{l}\text { European } \\
\text { Commission }\end{array}$ & 2012 & General & $x$ & - & Basado en evidencia \\
\hline 3 & $\begin{array}{l}\text { SADMFR Guidelines for the use of cone-beam compu- } \\
\text { ted tomography / Digital Volume Tomography (7) }\end{array}$ & SADMFR & 2015 & General & $x$ & - & Consenso \\
\hline 4 & $\begin{array}{l}\text { Oral Health Assessment and Review Dental Clinical } \\
\text { Guidance (8) }\end{array}$ & SDCEP & 2012 & General & - & $x$ & Basado en evidencia \\
\hline 5 & $\begin{array}{l}\text { Radiation Protection } 136 \text {. } \\
\text { The safe use of radiographs in dental practice (24) }\end{array}$ & $\begin{array}{l}\text { European } \\
\text { Commission }\end{array}$ & 2004 & General & - & $x$ & Basado en evidencia \\
\hline 6 & $\begin{array}{l}\text { Dental Radiographic Examinations: } \\
\text { Recommendations for patient selection and limiting } \\
\text { radiation exposure (9) }\end{array}$ & ADA-CSA & 2012 & General & - & $x$ & Juicio de expertos \\
\hline 7 & Position statement on use of CBCT in Endodontics (37) & AAE-AAOMR & 2015 & Endodoncia & $x$ & - & Basado en evidencia \\
\hline 8 & European Society of Endodontology position statement (38) & ESE & 2014 & Endodoncia & $x$ & - & Juicio de expertos \\
\hline 9 & $\begin{array}{l}\text { Position statement of the American Academy of Oral and } \\
\text { Maxillofacial Radiology on selection criteria for the use } \\
\text { of radiology in dental implantology with emphasis on } \\
\text { cone beam computed tomography (34) }\end{array}$ & Tyndall et al & 2012 & Implantología & $x$ & - & Juicio de expertos \\
\hline 10 & $\begin{array}{l}\text { E.A.0. guidelines for the use of diagnostic imaging in } \\
\text { implant dentistry. A consensus workshop organized by } \\
\text { the European Association for Osseointegration at the } \\
\text { Medical University of Warsaw (35) }\end{array}$ & Harris et al & 2012 & Implantología & $x$ & $x$ & Juicio de expertos \\
\hline \multirow[t]{2}{*}{11} & $\begin{array}{l}\text { Use of Cone Beam Computed Tomography in Implant } \\
\text { Dentistry: The International Congress of Oral Implantolo- } \\
\text { gists Consensus Report (39). }\end{array}$ & $\begin{array}{l}\text { Benavides } \\
\text { et al }\end{array}$ & 2012 & Implantología & $\mathrm{X}$ & - & Juicio de expertos \\
\hline & $\begin{array}{l}\text { American Academy of Periodontology Best Evidence } \\
\text { Consensus Statement on Selected Oral Applications for } \\
\text { Cone-Beam Computed Tomography (40) }\end{array}$ & $\begin{array}{l}\text { Mandelaris } \\
\text { et al }\end{array}$ & 2017 & Periodoncia & $x$ & - & Basado en evidencia \\
\hline 12 & The Good Practitioner's Guide to Periodontology (49) & BSP & 2016 & Periodoncia & $\mathrm{x}$ & - & Basado en evidencia \\
\hline 13 & $\begin{array}{l}\text { Guidelines for the use of radiographs in clinical or- } \\
\text { thodontics (36) }\end{array}$ & BOS & 2013 & Ortodoncia & $x$ & $x$ & Basado en evidencia \\
\hline 14 & $\begin{array}{l}\text { Clinical recommendations regarding use of CBCT in } \\
\text { orthodontics. Position statement by the AAOMR (41) }\end{array}$ & AAOMR & 2013 & Ortodoncia & $x$ & - & Consenso \\
\hline 15 & $\begin{array}{l}\text { Prescribing Dental Radiographs for Infants, Chil- } \\
\text { dren, Adolescents, and Individuals with Special } \\
\text { Health Care Needs (45) }\end{array}$ & AAPD & 2017 & Odontopediatría & - & $x$ & Basado en evidencia \\
\hline 16 & $\begin{array}{l}\text { Cone-beam CT in paediatric dentistry: DIMITRA pro- } \\
\text { ject position statement (22) }\end{array}$ & $\begin{array}{l}\text { Oenning } \\
\text { et al }\end{array}$ & 2018 & Odontopediatría & $x$ & - & Basado en evidencia \\
\hline 17 & $\begin{array}{l}\text { International Association of Dental Traumatology } \\
\text { guidelines for the management of traumatic dental } \\
\text { injuries (42-44) }\end{array}$ & IADT & 2012 & Trauma & - & $x$ & Juicio de expertos \\
\hline
\end{tabular}

Tabla2. Publicaciones identificadas como guías clínicas, con su año de publicación y áreas clínicas en las que se orientan sus directrices. Para la clasificación del método de desarrollo de la guía se consideró como Basado en evidencia cuando la guía lo mencionaba así, aún si utilizó metodología secundaria como consenso o juicio de expertos. Para la clasificación Consenso o Juicio de expertos se consideró las guías que lo mencionaban como único método de elaboración, sin utilización de evidencia.

FGDP Faculty of General Dental Practice. SADMFR Swiss Association of Dentomaxillofacial Radiology. SDCEP Scottish Dental Clinical Effectiveness Programme. ADA-CSA American Dental Association Council on Scientific Affairs. AAE-AAOMR American Association of Endodontists- American Academy of Oral and Maxillofacial Radiology. ESE European Society of Endodontology. BOS British Orthodontic Society. AAOMR American Academy of Oral and Maxillofacial Radiology. AAPD American Academy of Pediatric Dentistry. DIMITRA Dentomaxillofacial paediatric imaging: an investigation towards low dose radiation induced risks. IADT International Association of Dental Traumatology. 
clínicas que pueden ser útiles al momento de seleccionar el examen más apropiado para cada situación clínica. Las guías clínicas tienen como propósito entregar recomendaciones para el uso de distintas técnicas radiográficas. ${ }^{(29)}$

Las guías clínicas pueden desarrollarse en base a tres enfoques; uno es mediante el juicio de expertos, otro es mediante un consenso, y el tercero es usar una metodología basada en evidencia. ${ }^{(30)}$ Las guías clínicas basadas en evidencia se realizan en base a revisiones sistemáticas de la literatura, considerando la calidad de la evidencia revisada. Es por esta metodología que las guías clínicas basadas en evidencia poseen menor posibilidad de sesgo al tener menor influencia de las opiniones individuales. $^{(29)}$

Las guías clínicas no son reglamentos rígidos, sino más bien, un criterio de orientación para la elección del examen radiográfico. ${ }^{(5)}$ Estas guías incluyen aspectos generales del uso de exámenes radiográficos en odontología y diversas indicaciones clínicas de la práctica diaria(7) que facilitan al clínico el identificar el examen beneficioso para cada paciente. ${ }^{(31)}$ La guía debe considerarse una ayuda en el proceso de justificación del examen radiográfico, en que cada caso se evalúe de manera específica, según la necesidad individual de cada paciente, realizando una historia clínica y médica completa. ${ }^{(5-7)}$

Las guías clínicas ayudan al clínico a poner en práctica la odontología basada en evidencia, mediante la toma de decisiones clínicas que signifiquen una atención de calidad y mayor beneficio para el paciente. ${ }^{\left({ }^{32}\right)}$ Esto se logra cuando el clínico considera las necesidades y preferencias del paciente para su tratamiento y las relaciona con las recomendaciones que entregan las guías, integrando esta información con su experiencia clínica. ${ }^{(33)}$

Existen numerosas guías clínicas tanto para los exámenes radiográficos convencionales bidimensionales como para TCHC. En el caso de la TCHC, la evidencia de estas guías se limita a situaciones clínicas específicas, existiendo poca evidencia respecto de la eficacia diagnóstica en los niveles superiores del modelo de Fryback y Thornbury. ${ }^{(5,}$ 28 ,29) La mayoría de la evidencia que aportan las guías para TCHC está limitada a los dos niveles inferiores de este modelo. ${ }^{(5)}$
Diferentes asociaciones del mundo han planteado recomendaciones para el uso de los exámenes radiográficos en odontología. ${ }^{(34-45)}$. Estas recomendaciones se enfocan en exámenes 2D, 3D, o ambos ${ }^{(6,34-36)} ; \mathrm{y}$ pueden cubrir todas las áreas de la odontología ${ }^{(5-9,24)}$ o ser específicas para las distintas especialidades. ${ }^{(34-45)}$

En el caso de la implantología, por ejemplo, se ha intentado unificar criterios y establecer recomendaciones para el uso adecuado de la TCHC en la evaluación pre-implantológica, basándose en las guías clínicas. ${ }^{(46,47)}$ En esta área se ha encontrado que la mayoría de guías clínicas para implantes basan sus recomendaciones en consenso, sin base en evidencia científica, ${ }^{(46)}$ existiendo diferencias en sus recomendaciones. ${ }^{(29)}$ En el caso de los exámenes $2 \mathrm{D}$, una revisión sistemática reciente sobre guías para el uso de las radiografías bitewing en el diagnóstico de caries dental, encontró diferencias en las recomendaciones en cuanto a la edad en que debe tomarse la primera radiografía, y el intervalo entre la toma de radiografías. ${ }^{(48)}$

La ausencia de criterios definidos y basados en evidencia podría llevar a la existencia de indicaciones de exámenes no justificados, lo que resalta la importancia de enfatizar la aplicación del principio de justificación en odontología.

La Tabla 2 muestra guías clínicas para el uso de exámenes radiográficos en odontología, con su año de publicación y áreas clínicas en las que se orientan sus recomendaciones.

Una correcta aplicación de las recomendaciones de guías basadas en evidencia podría reflejarse en una reducción de dosis de radiación en los pacientes, al evitar exposiciones innecesarias. (6) Recientes investigaciones sobre los usos de la $\mathrm{TCHC}^{(50-54)}$ han encontrado que la mayoría de las indicaciones de exámenes de TCHC están en línea con las recomendaciones de la Guía 172 de la Comisión Europea. ${ }^{(5)}$ Esto podría demostrar el impacto positivo de las guías clínicas y la correcta aplicación del principio de justificación de los exámenes de TCHC.

\section{CONCLUSIONES}

Es necesario que tanto pacientes como clínicos conozcan el riesgo que supone la exposición a la 
radiación, para que se entienda la necesidad y la importancia de la aplicación del principio de justificación. El uso de guías clínicas y la comunicación entre el clínico y el radiólogo maxilofacial son fundamentales para lograr con éxito el proceso de justificación. Es necesaria más investigación en los niveles superiores del modelo de Fryback y Thornbury, que permitan justificar la indicación de nuevas tecnologías radiográficas en odontología. Esta justificación se produciría al demostrar el impacto de la nueva tecnología radiográfica en el diagnóstico y/o plan de tratamiento del paciente. Investigación realizada en niveles superiores de eficacia también contribuiría al desarrollo de guías clínicas basadas en evidencia.

\section{BIBLIOGRAFIA}

1. INTERNATIONAL COMMISSION ON RADIOLOGICAL PROTECTION. The 2007 Recommendations of the International Commission on Radiological Protection. 2007; ICRP Publication 103. Ann ICRP, 37.

2. INTERNATIONAL COMMISSION ON RADIOLOGICAL PROTECTION. Radiological Protection in Cone Beam Computed Tomography (CBCT). 2015; ICRP Publication 129. Ann ICRP 44.

3. FDI WORLD DENTAL FEDERATION. FDI policy statement on radiation safety in dentistry: adopted by the FDI General Assembly. Int Dent J 2014;64:289-90.

4. INTERNATIONAL COMMISSION ON RADIOLOGICAL PROTECTION. Ethical Foundations of the System of Radiological Protection. 2018; ICRP Publication 138. Ann ICRP 47.

5. EUROPEAN COMMISSION. Radiation Protection $N^{\circ}$ 172: Cone beam CT for dental and maxillofacial radiology. Evidence-based guidelines. 2012 [citado 11 de junio de 2018]. http://www.sedentexct.eu/files/radiation_protection_172.pdf

6. Horner K, Eaton KA. Selection Criteria for Dental Radiography. 3.a ed. London, UK: Faculty of General Dental Practice UK; 2013.

7. Dula K, Benic GI, Bornstein M, Dagassan-Ber- ndt D, Filippi A, Hicklin S, et al. SADMFR Guidelines for the use of cone-beam computed tomography/digital volume tomography. Swiss Dent J 2015;125:945-53.

8. Scottish Dental Clinical Effectiveness Programme. Oral Health Assessment and Review Dental Clinical Guidance. 2012.

9. American Dental Association Council on Scientific Affairs. Dental radiograph examinations: recommendations for patient selection and limiting radiation exposure. US Department of Health and Human Services, Public Health Service, Food and Drug Administration. 2012 [citado 29 de agosto de 2018]. https:// www.ada.org/ /media/ADA/Member\%20 Center/Flles/Dental_Radiographic_Examinations_2012.ashx

10. Fryback DG, Thornbury JR. The efficacy of diagnostic imaging. Med Decis Mak Int J Soc Med Decis Mak 1991;11:88-94.

11. White SC, Pharoah MJ, editores. Oral radiology: principles and interpretation. Edition 7. St. Louis, Missouri: Elsevier; 2014.

12. White SC, Scarfe WC, Schulze RKW, Lurie AG, Douglass JM, Farman AG, et al. The Image Gently in Dentistry campaign: promotion of responsible use of maxillofacial radiology in dentistry for children. Oral Surg Oral Med Oral Pathol Oral Radiol 2014;118:257-61.

13. Widder J. The origins of radiotherapy: discovery of biological effects of X-rays by Freund in 1897, Kienböck's crucial experiments in 1900 , and still it is the dose. Radiother Oncol J Eur Soc Ther Radiol Oncol 2014;112:150-2.

14. Sansare K, Khanna V, Karjodkar F. Early victims of X-rays: a tribute and current perception. Dentomaxillofac Radiol 2011;40:123-5.

15. Solano JB, Gómez de la Cámara A, García F, Fernández A. Efectos biológicos de la exposición a dosis bajas de radiación ionizante. Madrid: Asociación Española de la Industria Eléctrica UNESA; 2009.

16. INTERNATIONAL COMMISSION ON RADIOLOGICAL PROTECTION. Statement on Tissue 
Reactions. 2011 [citado 21 de septiembre de 2018]. http://www.icrp.org/page.asp?id=123

17. Hedesiu M, Marcu M, Salmon B, Pauwels $\mathrm{R}$, Oenning $\mathrm{AC}$, Almasan $\mathrm{O}$, et al. Irradiation provided by dental radiological procedures in a pediatric population. Eur $\mathrm{J}$ Radiol 2018;103:112-7.

18. Kalender W.A. Computed Tomography: Fundamentals, System Technology, Image Quality, Applications. Munich: Publicis Publishing; 2011.

19. National Council on Radiation Protection and Measurements. Commentary No. 27 - Implications of Recent Epidemiologic Studies for the Linear-Nonthreshold Model and Radiation Protection. 2018 [citado 13 de agosto de 2018]. https://ncrponline.org/wp-content/ themes/ncrp/Pub_announcements/Commentary_No27_overview.pdf

20. INTERNATIONAL COMMISSION ON RADIOLOGICAL PROTECTION. Recommendations of the ICRP. 1977; ICRP Publication 26. Ann ICRP 1.

21. Bushberg JT. Eleventh annual Warren K. Sinclair keynote address-science, radiation protection and NCRP: building on the past, looking to the future. Health Phys 2015;108:115-23.

22. Oenning AC, Jacobs $R$, Pauwels $R$, Stratis $A$, Hedesiu M, Salmon B, et al. Cone-beam CT in paediatric dentistry: DIMITRA project position statement. Pediatr Radiol 2018;48:308-16.

23. Hidalgo A, Theodorakou C, Horner K. Protección radiológica en tomografía computarizada Cone-Beam en odontología. Anu Soc Radiol Oral Maxilo Facial Chile 2013;16:23-32.

24. EUROPEAN COMMISSION. European guidelines on radiation protection in dental radiology: the safe use of radiographs in dental practice. 2004 [citado 10 de septiembre de 2018]. https://ec.europa.eu/energy/sites/ener/files/ documents/136.pdf

25. INTERNATIONAL ATOMIC ENEGRY AGENCY. Comprehensive Clinical Audits of Diagnostic Radiology Practices: A Tool for Quality Improvement Quality Assurance Audit for Diagnostic Radiology Improvement and Learning (QUAADRIL). 2011 [citado 10 de diciembre de 2018]. http://www-pub.iaea.org/ books/IAEABooks/8613/Comprehensive-Clinical-Audits-of-Diagnostic-Radiology-Practices-A-Tool-for-Quality-Improvement-Quality-Assurance-Audit-for-Diagnostic-Radiology-Improvement-and-Learning-QUAADRIL

26. Oswal D, Sapherson D, Rehman A. A study of adequacy of completion of radiology request forms. Radiography 2009;15:209-13.

27. Ramírez J, Rodríguez C, Quiroz O, Motta G. La comunicación del radiólogo con médicos tratantes y pacientes. Acta med. Grupo Angeles 2007;5:228-232.

28. Geist JR. The efficacy of diagnostic imaging should guide oral and maxillofacial radiology research. Oral Surg Oral Med Oral Pathol Oral Radiol 2017;124:211-3.

29. Horner K, O'Malley L, Taylor K, Glenny A-M. Guidelines for clinical use of CBCT: a review. Dentomaxillofacial Radiol 2015;44:20140225.

30. Kwong JSW, Chen H, Sun X. Development of Evidence-based Recommendations: Implications for Preparing Expert Consensus Statements. Chin Med J (Engl) 2016;129:29983000 .

31. Schulze RKW. Editorial: guidelines for oral and maxillofacial radiology. Dentomaxillofacial Radiol 2016;45.

32. Gonçalves APR, Correa MB, Nahsan FPS, Soares CJ, Moraes RR. Use of scientific evidence by dentists in Brazil: Room for improving the evidence-based practice. PloS One 2018;13:e0203284.

33. FDI WORLD DENTAL FEDERATION. FDI policy statement on Evidence-based dentistry: Adopted by the FDI General Assembly, September 2016, Poznan, Poland. Int Dent J 2017;67:12-3.

34. Tyndall DA, Price JB, Tetradis S, Ganz SD, Hildebolt C, Scarfe WC, et al. Position statement of the American Academy of Oral and Maxillofacial Radiology on selection criteria 
for the use of radiology in dental implantology with emphasis on cone beam computed tomography. Oral Surg Oral Med Oral Pathol Oral Radiol 2012;113:817-26.

35. Harris D, Horner K, Gröndahl K, Jacobs R, Helmrot E, Benic GI, et al. E.A.O. guidelines for the use of diagnostic imaging in implant dentistry 2011. A consensus workshop organized by the European Association for Osseointegration at the Medical University of Warsaw. Clin Oral Implants Res 2012;23:1243-53.

36. Isaacson KG, Thom AR, Atack NE, Horner K, Whaites E. Guidelines for the Use of Radiographs in Clinical Orthodontics. 4.a ed. Vol. 31. London, UK: British Orthodontic Society; 2015.

37. Special Committee to Revise the Joint AAE/ AAOMR Position Statement on use of CBCT in Endodontics. AAE and AAOMR Joint Position Statement: Use of Cone Beam Computed Tomography in Endodontics 2015 Update. Oral Surg Oral Med Oral Pathol Oral Radiol 2015;120:508-12.

38. European Society of Endodontology, Patel S, Durack C, Abella F, Roig M, Shemesh H, et al. European Society of Endodontology position statement: the use of CBCT in endodontics. Int Endod J 2014;47:502-4.

39. Benavides E, Rios HF, Ganz SD, An C-H, Resnik R, Reardon GT, et al. Use of cone beam computed tomography in implant dentistry: the International Congress of Oral Implantologists consensus report. Implant Dent 2012;21:78-86.

40. Mandelaris GA, Scheyer ET, Evans M, Kim D, McAllister B, Nevins ML, et al. American Academy of Periodontology Best evidence consensus statement on selected oral applications for cone-beam computed tomography. J Periodontol 2017;88:939-45.

41. American Academy of Oral and Maxillofacial Radiology. Clinical recommendations regarding use of cone beam computed tomography in orthodontics. Position statement by the American Academy of Oral and Maxillofacial Radiology. Oral Surg Oral Med Oral Pathol
Oral Radiol 2013;116:238-57.

42. Diangelis AJ, Andreasen JO, Ebeleseder KA, Kenny DJ, Trope M, Sigurdsson A, et al. International Association of Dental Traumatology guidelines for the management of traumatic dental injuries: 1. Fractures and luxations of permanent teeth. Dent Traumatol Off Publ Int Assoc Dent Traumatol 2012;28:2-12.

43. Andersson L, Andreasen JO, Day P, Heithersay G, Trope M, Diangelis AJ, et al. International Association of Dental Traumatology guidelines for the management of traumatic dental injuries: 2. Avulsion of permanent teeth. Dent Traumatol Off Publ Int Assoc Dent Traumatol 2012;28:88-96.

44. Malmgren B, Andreasen JO, Flores MT, Robertson A, DiAngelis AJ, Andersson L, et al. International Association of Dental Traumatology guidelines for the management of traumatic dental injuries: 3. Injuries in the primary dentition. Dent Traumatol Off Publ Int Assoc Dent Traumatol 2012;28:174-82.

45. American Academy of Pediatric Dentistry. Prescribing dental radiographs for infants, children, adolescents, and individuals with special health care needs. Pediatr Dent 2017;39:205-7.

46. Bornstein MM, Scarfe WC, Vaughn VM, Jacobs $\mathrm{R}$. Cone beam computed tomography in implant dentistry: a systematic review focusing on guidelines, indications, and radiation dose risks. Int J Oral Maxillofac Implants 2014;29 Suppl:55-77.

47. Carrasco Meza A, Quintanilla Sfeir M, Hidalgo Rivas A. Guías sobre el uso de tomografía computarizada de haz cónico en la evaluación pre-quirúrgica en implantología. Av En Odontoestomatol 2018;34:183-92.

48. Goodwin TL, Devlin H, Glenny AM, O'Malley $\mathrm{L}$, Horner K. Guidelines on the timing and frequency of bitewing radiography: a systematic review. Br Dent J 2017;222:519-26.

49. British Society of Periodontology. BSP Good Practitioner's Guide to Periodontology. 2016. [citado 28 de marzo de 2019]. https://www. bsperio.org.uk/publications/good_practitio- 
ners_guide_2016.pdf?v=3

50. Barba L, Berrocal AL, Hidalgo A. Uses of cone-beam computed tomography in San José, Costa Rica. Imaging Sci Dent 2018;48:103-9.

51. Arancibia B, Schilling J, Schilling A, Correa-Beltrán G, Hidalgo A. Usos de tomografía computarizada de haz cónico en menores de 25 años en Talca, Chile. Rev Cuba Estomatol 2017;54.

52. Isman Ö, Yılmaz HH, Aktan AM, Yilmaz B. Indications for cone beam computed tomography in children and young patients in a Turkish subpopulation. Int $\mathrm{J}$ Paediatr Dent 2017;27:183-90.

53. Van Acker JWG, Martens LC, Aps JKM. Co- ne-beam computed tomography in pediatric dentistry, a retrospective observational study. Clin Oral Investig 2016;20:1003-10.

54. Hidalgo-Rivas JA, Theodorakou C, Carmichael F, Murray B, Payne M, Horner K. Use of cone beam $\mathrm{CT}$ in children and young people in three United Kingdom dental hospitals. Int $\mathrm{J}$ Paediatr Dent 2014;24:336-48.

\section{CORRESPONDENCIA:}

Alejandro Hidalgo Rivas

Escuela de Odontología

Universidad de Talca

Avenida Lircay S/N

Talca, Chile

Teléfono +56-71-2200476

Correo electrónico: ahidalgo@utalca.cl 